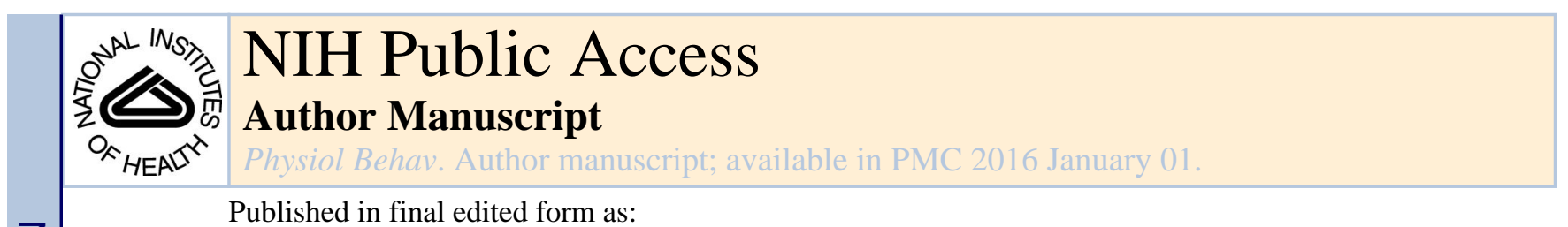

Published in final edited form as:

Physiol Behav. 2015 January ; 0: 124-132. doi:10.1016/j.physbeh.2014.10.022.

\title{
A Pilot Randomized Trial of a Cognitive Reappraisal Obesity Prevention Program
}

\author{
Eric Stice, $\mathrm{PhD}^{1}$, Sonja Yokum, $\mathrm{PhD}^{1}$, Kyle Burger, $\mathrm{PhD}^{2}$, Paul Rohde, $\mathrm{PhD}^{1}$, Heather Shaw, \\ $\mathbf{P h D}^{1}$, and Jeff M. Gau, MS ${ }^{1}$ \\ ${ }^{1}$ Oregon Research Institute \\ ${ }^{2}$ University of North Carolina at Chapel Hill
}

\begin{abstract}
Background/Objectives-Evaluate a selective obesity prevention program promoting use of cognitive reappraisals to reduce reward region response and increase inhibitory region response to high-fat/high-sugar foods and reduce intake of fat and sugar to prevent blunted reward region response to intake of such foods.
\end{abstract}

Subjects/Methods-Young adults at risk for future weight gain by virtue of weight concerns $(N$ $=148$ ) were randomized to this new prevention program (Minding Health), an alternative prevention program promoting participant-driven gradual reductions in caloric intake and increases in physical activity (Healthy Weight), or an obesity education video control condition, completing assessments at pre, post, and 6-month follow-up. A subset of Minding Health and control participants completed an fMRI scan at pre and post assessing neural response to images of high-fat/sugar foods and to receipt and anticipated receipt of a high-fat/sugar food.

Results-Minding Health participants showed significantly greater reductions in body fat than controls and caloric intake from fat and sugar than Healthy Weight participants. However, Healthy Weight participants showed greater reductions in BMI and eating disorder symptoms than Minding Health participants and Minding Health participants showed greater activation of an inhibitory control region and reduced activation of an attention/expectation region in response to palatable food images relative to pretest and controls.

Conclusions-Although the Minding Health intervention produced some of the hypothesized effects, it did not produce lasting reductions in body fat or BMI and showed limited effects on neural responsivity, implying it will be vital to increase the efficacy of this new prevention program.

(C) 2014 Elsevier Inc. All rights reserved.

Dr. Stice is the corresponding author. He can be contacted at: 1776 Millrace Drive, Eugene Oregon 97403, Phone: 541-484-2123, Fax: 541-484-1108, estice@ori.org.

The authors report no conflict of interest with respect to the content of this paper.

Publisher's Disclaimer: This is a PDF file of an unedited manuscript that has been accepted for publication. As a service to our customers we are providing this early version of the manuscript. The manuscript will undergo copyediting, typesetting, and review of the resulting proof before it is published in its final citable form. Please note that during the production process errors may be discovered which could affect the content, and all legal disclaimers that apply to the journal pertain. 


\section{Keywords}

prevention; obesity; weight gain; fMRI; reward regions; inhibitory regions

Nearly $70 \%$ of US adults are overweight or obese, which is credited with 300,000 deaths and $\$ 150$ billion in health-related expenses yearly (1). However, obesity prevention programs have not produced clinically meaningful reductions in weight gain (2). Thus, a public health priority is to design more effective prevention programs. We submit that findings from brain imaging studies on neural responsivity that predicts weight gain, and strategies to reduce this aberrant responsivity, may guide the design of more effective prevention programs.

\section{Elevated Brain Reward Region Responsivity to Food}

Obese versus lean humans show greater reward region responsivity (striatum, amygdala, orbitofrontal cortex [OFC], insula) to high-fat/sugar food images (3-6). Critically, elevated amygdala response to high-fat/sugar food images (7), ventral striatum response to high-fat/ sugar food images (8), and OFC response to cues signaling impending presentation of highfat/sugar food images predicted future weight gain (9). Thus, obese versus lean humans show greater reward region activation as they encounter unhealthy food images/cues and this increases risk for weight gain. Results support the thesis that reward region hyperresponsivity increases risk for overeating (10) and incentive salience theory (11), which posits that food images/cues activate reward circuitry through conditioned associations with rewarding food intake, eventually producing cravings that contribute to overeating.

\section{Lower Inhibitory Region Responsivity to Food}

Obese versus lean humans show less activation of prefrontal regions (medial frontal gyrus and ventrolateral prefrontal cortex [vlPFC]) when trying to inhibit responses to unhealthy food images and more "go" responses on no-go unhealthy food trials (12). They also show less activation in inhibitory control regions in response to unhealthy food images $(10,13)$ and food logos (14), as well as a preference for immediate food reward over larger delayed food rewards and behavioral response inhibition deficits on non-food go/no-go and stopsignal tasks (15-17). Lower dorsolateral prefrontal cortex (dlPFC) responsivity to unhealthy food images predicted increased objectively measured food intake over 3 days (18) and less activation of prefrontal and executive regions (inferior, middle, superior frontal gyri) during delayed discounting choices predicted greater weight gain (19). Individuals with less inhibitory control also show greater weight gain $(20,21)$ and poorer response to obesity treatment (22). Thus, obese individuals show less recruitment of inhibitory regions in response to food cues and lower activation of inhibitory control regions predicts weight gain.

\section{Top-down Control of Reward and Inhibitory Region Responsivity to Food}

Cognitive reappraisals, such as thinking of long-term health consequences of eating unhealthy food when viewing images of such foods, has been found to increase inhibitory 
region (dlPFC, vlPFC, lateral OFC, superior and inferior frontal gyri) activation and decrease reward region (ventral striatum, amygdala, ACC, midbrain) and attention region (precuneus, posterior cingulate cortex) activation (23-26). Stoeckel and associates (27) used real-time fMRI biofeedback to augment the effects of cognitive reappraisals in reducing reward region responsivity and increasing inhibitory region responsivity to palatable food images; training resulted in greater reduction in medial OFC, ventral striatum, and amygdala, and greater inhibitory control region activation (inferior frontal cortex [IFG]) in response to palatable food images. Thus, cognitive reappraisal trainings may reduce hyperresponsivity of reward regions to food cues and increase inhibitory control region activation, which is crucial because our environment is replete with food images/cues, including TV food ads (2) that contribute to overeating unhealthy foods $(2,28)$.

Accordingly, we developed an obesity prevention program that trained participants to use cognitive reappraisals when confronted with unhealthy tempting foods. We hypothesize that if participants learn to automatically apply these cognitive reappraisals, they will show reduced reward and attention region responsivity and increased inhibitory region responsivity to food images and cues signaling impending delivery of a high-fat/high-sugar food, which should result in reduced caloric intake.

\section{Decreased Reward Region Responsivity to Palatable Food Intake}

Paradoxically, obese versus lean humans have fewer striatal dopamine (DA) receptors (29, 30) and show less striatal response to high-fat/sugar beverage intake (10,31-33), consistent with the thesis that people overeat to compensate for a reward deficit (30). Yet it is unclear whether reduced reward region response to food intake is an initial vulnerability factor or a result of overeating. Women who gained weight showed a reduced striatal response to milkshake receipt relative to baseline and weight stable women (34). Rats randomized to overeating versus control conditions show down-regulation of post-synaptic D2 receptors, and reduced D2 sensitivity, extracellular DA levels in the nucleus accumbens and DA turnover, and lower sensitivity of DA reward circuitry to food intake, electrical stimulation, and amphetamine administration $(35,36)$. A 5-week high-fat/sugar diet versus isocaloric intake of a low-fat/sugar diet resulted in down-regulation of striatal D1 and D2 receptors in rats, implying that energy-dense food intake (37), rather than positive energy balance, induces reward neuroplasticity. Results suggest that regular intake of high-fat/sugar food reduces reward region responsivity to such foods.

If overeating energy-dense food reduces reward region response to such food, which prompts compensatory overeating, reducing fat and sugar intake may help people avoid this induced-reward deficit. Such a "palate-retraining" intervention may also reduce preferences for high-fat/sugar foods, which may contribute to weight gain. Reducing intake of dietary fat decreases preferences and frequency of future consumption of previously preferred high-fat foods and increases acceptance of low-fat foods $(38,39)$. Chronic intake of a high-fat diet theoretically leads to reduced oral sensitivity, prompting compensatory escalations in fat intake to experience comparable reward (40). We therefore included a palate-retraining component to our obesity prevention program wherein participants reduce fat and sugar intake to decrease preferences for these foods and avoid reduced reward region responsivity 
to high-fat/sugar food intake observed in obese humans. We hypothesize that if intervention participants reduce consumption of fat and sugar, they will show an increased striatal response to receipt of a high-fat/high-sugar milkshake, which may reduce risk for overeating.

The aims of this pilot were to evaluate the effects of a new obesity prevention program involving training in cognitive reappraisals and palate retraining (the Minding Health intervention) to an alternative prevention program promoting participant-driven gradual reductions in caloric intake and increases in physical activity (the Healthy Weight intervention) and an educational video control condition. A subset of Minding Health and control participants completed an fMRI paradigm assessing neural response to palatable food images and to receipt and anticipated receipt of palatable food.

\section{Methods}

\section{Participants and Procedure}

Participants were 148 young adults ( $M$ age $=19.3 \pm 1.2 ; 72 \%$ female $)$ with a mean baseline BMI of $23.5 \pm 2.7$. We focused on first-year college students because they are at risk for weight gain (41). The sample was 3\% Native American, 10\% Asian, 6\% African American, and $81 \%$ European American. Participants were recruited using mailings, flyers, and leaflets inviting students with weight concerns to participate in a weight control trial. Informed consent was obtained for this IRB-approved trial. Participants had to affirm that they had weight concerns. The sole exclusion criterion was a current DSM-IV anorexia nervosa, bulimia nervosa, or binge eating disorder. Participants were randomized to the Minding Health intervention, the Healthy Weight intervention, or an obesity education video control condition. This pilot was an add-on to a larger obesity prevention trail comparing the efficacy of the Healthy Weight obesity prevention program to an educational video control condition, which is why a fewer participants were assigned to the new Minding Health obesity prevention program. Figure 1 provides participant flow information. Minding Health was delivered in 7 1-hr weekly group meetings and Healthy Weight in 61 -hr weekly group meetings to groups of 6-10 participants by 2 clinicians. We made the Minding Health intervention one session longer than the Healthy Weight intervention because we thought it necessary to proved sufficient massed-practice of the cognitive reappraisals. If a participant missed a session, a 10-15 minute individual session was conducted to review missed material when possible. Facilitators read the intervention script and attended a 4-hour workshop to role-play key session elements and discuss process issues.

Participants provided interview and survey data at pre, post, and 6-month follow-up. Female assessors blind to condition attended 24 hours of training, wherein they received instruction in diagnostic interview skills, observed simulated interviews, and role-played interviews. They had to demonstrate inter-rater agreement (kappa $[k]>.80$ ) with supervisors using 12 tape-recorded interviews before collecting data. Participants received $\$ 30$ for completing each assessment. Those who completed both fMRI scans were paid an additional $\$ 150$.

Minding Health-Session 1 began with a brief rapport-building exercise and an overview of the conceptual intervention foundations. Participants then learned to use cognitive 
reappraisals to reduce desire for and intake of unhealthy foods, and generated several reappraisals. In sessions 2-6 they practiced generating reappraisals when exposed to pictures and slides of unhealthy foods. In some exercises they generated reappraisals rapidly to promote automatic use of this strategy. Participants were also encouraged to use reappraisals to increase consumption of healthy foods (fruits and vegetables), such as thinking of the long-term health benefits of eating healthy foods. In sessions 6 and 7 participants practiced generating reappraisals in response to real unhealthy and healthy foods. For the unhealthy foods, they articulated a health cost of eating the food (e.g., a snickers), opened it and tossed it in the garbage. For the healthy foods, they articulated a health benefit of eating the food (e.g., baby carrots), and then could eat the food. Participants were asked to apply the reappraisal strategies between sessions and to share how that went. They were also encouraged to gradually reduce dietary intake of fat and sugar though a participant-driven dietary modification plan. Sessions began with a brief motivational enhancement exercise involving participant-led discussions of the (a) costs of obesity and an unhealthy diet, (b) benefits of a healthy weight and a healthy diet and (c) benefits of using the intervention strategies.

Healthy Weight-Session 1 began with a brief rapport-building exercise and an overview of the conceptual intervention foundations. To improve motivation, participants discussed health, social, and emotional benefits of pursuing the healthy ideal. They were then told that making gradual healthy lifestyle improvements to dietary intake and physical activity helps balance caloric intake with expenditure and people achieve the healthy ideal. Participants identified healthy changes they could make to their dietary intake and activity level over the next week (e.g., have a daily salad and exercise 3 times a week for 30 mins). For homework, participants were asked to make the personalized lifestyle improvements and to complete diet and exercise logs. Sessions 2 through 4 focused on discussing the participant-driven lifestyle improvement plans, providing group support, and planning additional ways to improve dietary intake and physical activity on an iterative basis. Participants were asked to reward themselves for making positive lifestyle improvements. Additional topics, such as the importance of avoiding extended periods of caloric restriction, were discussed. In session 5 a slip response plan was discussed and participants were encouraged to focus on maintaining their healthy lifestyle improvements and were asked to cook a healthy meal or exercise with a friend as a home exercise. In session 6 participants described how the home exercises went, discussed maintaining their healthy lifestyle behavior changes over the next 6 months, and completed a closure exercise in which they acknowledged the gains they had made.

Educational video condition-Participants were asked to view Weight of the World, a 51-min documentary on obesity, with a focus on the health consequences of excess body weight, and the contributions made by the food industry, labor saving technologies, and urban sprawl.

\section{Measures}

Body fat-We used air displacement plethysmography (ADP) via the Bod Pod S/T to assess percent body fat. ADP estimates of percent body fat show high test-retest reliability ( $r$ 
$=.92-.99)$, correlate with DEXA and hydrostatic weighing estimates $(r=.98-.99)$, and have a mean difference of only $1.7 \%$ relative to DEXA estimates (42).

Body mass-Raw BMI scores reflected change in height-adjusted weight over follow-up because these scores are superior to age- and sex-adjusted percentiles or BMIz scores for modeling change over time in longitudinal data (43). After removing shoes and coats, height was measured to the nearest millimeter using stadiometers and weight was assessed to the nearest $0.1 \mathrm{~kg}$ using digital scales. BMI correlates with health measures, such as blood pressure, adverse lipoprotein profiles, and diabetes mellitus (44).

Eating pathology-The Eating Disorder Diagnostic Interview, a semi-structured interview, assessed DSM-IV eating disorder symptoms. Items assessing symptoms in the past month were summed to form a composite, which has shown internal consistency ( $a=$. 92), 1-week test-retest reliability $(r=.90)$, inter-rater agreement $(r=.93)$, and sensitivity to detecting eating disorder prevention program effects (45).

Dietary Intake-Seventy-six items from the Block Food Frequency Questionnaire (BFFQ; (46) assessed frequency of consumption of various foods. Participants were given a definition of a medium portion and asked to indicate the frequency of consumption over the past 2 weeks. The USDA National Nutrient Database for Standard Reference software (http://ndb.nal.usda.gov/) was used by a registered dietitian to estimate percentage of calories consumed from fat and sugar (e.g., [(calories from fat + calories from sugar)/total calories]*100) because Minding Health encouraged participants to reduce intake of these macronutrients. BFFQ values correlated with 4-day food record estimates of energy intake ( $\mathrm{M} r=.57$; (46), were within $+/-3 \%$ of values obtained from 7-day weighed food records (47), and have shown 2-week test-retest reliability ( $\mathrm{M} \mathrm{r}=.69$ (48).

fMRI paradigms and analysis-A subset of Minding Health $(\mathrm{n}=7 ; 57 \%$ females $)$ and control participants ( $\mathrm{n}=9 ; 78 \%$ females) underwent scans at pre and post. They were asked to consume their regular meals, but refrain from eating or drinking caffeinated beverages for 4 hours preceding their scan. Order of the fMRI paradigms was counterbalanced (as were runs within paradigms). Upon arrival to their session participants rated their hunger on a scale from 1 (not hungry at all) to 10 (extremely hungry).

The event-related food picture paradigm (Fig 2a) assessed blood oxygen level dependent (BOLD) activation in response to pictures of high-fat/high-sugar foods $(n=30)$, vegetables $(n=30)$, and water glasses $(n=30)$. Images were presented for 5 seconds followed by a jitter (3.5-5 seconds) during which a fixation cross was presented. Participants were asked to pay attention to the pictures, but were not asked to apply cognitive reappraisals during exposure to either unhealthy or healthy foods because we wanted to provide an unbiased test of whether they automatically used reappraisals. The event-related milkshake paradigm (Fig $2 b$ ) assessed BOLD response to receipt and anticipated receipt of milkshake and tasteless solution. Stimuli were 2 cues (glasses of milkshake and water, 30 repeat each) that signaled impending delivery of either a chocolate milkshake or tasteless solution (30 repeat each). Cues were presented for 2 seconds, followed by a jitter (1-7 seconds) during which time the screen was blank. Taste delivery (5 seconds) occurred 7-9 seconds after cue onset. 
Participants were instructed to swallow during the 'swallow' cue (1 second). The next cue appeared 1-7 seconds after the 'swallow' cue went off.

Individual maps were constructed to compare activations within each participant for four events: 1) viewing pictures (duration 5 seconds) of high-fat/high-sugar food versus vegetables (energy-dense foods > vegetables), 2) viewing pictures of high-fat/high-sugar food versus glasses of water (energy-dense food > water), 3) viewing the cue (duration 2 seconds) signaling milkshake delivery versus that signaling tasteless solution (milkshake cue $>$ tasteless solution cue), 4) delivery (onset 7-9 seconds after cue onset; duration 5 seconds) of milkshake versus tasteless solution (milkshake receipt $>$ tasteless solution receipt). We conducted a 2 Group (intervention, control) $\times 2$ Time (pre, post) repeated-measures ANOVA on BOLD responses, using sex and hunger as covariates. Whole brain analyses were used throughout; activity surviving a threshold of $p<0.005$ and a cluster $(k) \geq 25$ was considered significant. This threshold is an overall significance level of $P<0.05$, corrected for multiple comparisons across the whole brain. Effect sizes $(r)$ were derived from the Zvalues $(Z / \downarrow N)$. Stice et al. $(34,49)$ provide greater details about the fMRI paradigm, data acquisition, and preprocessing.

Statistical Methods-Multiple imputation was used to replace missing values following best-practice recommendations (50). Missing data were imputed using IVEWare (51), which uses all available data to impute missing data via a sequential regression approach. Missing data was replaced with imputed data in 20 data sets, which were analyzed separately. Model parameters and standard errors, which incorporate within and between model parameter variability, were combined following Rubin (52) as implemented in SAS PROC MIANALYZE (SAS Institute Inc., 2011).

Of the 148 participants $97 \%$ completed all three assessments, $1 \%$ completed two, and $2 \%$ completed one assessment. Number of completed assessments was not related to condition $\left(\chi^{2}[4,148]=7.20, p=.13\right)$. Number of group sessions attended for Minding Health $(M=5.1$, $S D=2.3)$ and Healthy Weight $(M=5.4, S D=1.5)$ participants were similar $(t[84]=0.66, p=.51)$. Eating disorder symptoms were normalized with a log base ten transformations. We tested whether conditions differed on pretest outcomes and demographics (sex, race, ethnicity, age, parental education). No differences were found with the exception of eating disorder symptom scores $(F[2,147]=3.46, p=.034]$; Healthy Weight participants had significantly higher symptoms at pretest than Minding Health participants ( $M=12.6$ vs. 7.3, respectively).

Random effects growth models in a hierarchical linear model framework were fit with SAS PROC MIXED. We fit unconditional mixed models with person nested within group. The proportion of variance explained at each level of the model was computed, where level-1 units were the measurement occasion, which were nested with the level-2 unit (person), which was nested within the level-3 unit (group). There was minimal variance attributable to the group level: variability in the group random intercept was not significant for any outcome. Thus, level-3 group random effects were eliminated from models. Next, individual variability in change in study outcomes from posttest to 6-month follow-up was nested within individual and modeled as a function of condition. Models controlled for pretest 
values of the outcome. We conducted three planned contrasts: Minding Health versus controls, Minding Health versus Healthy Weight, and Healthy Weight versus controls (consistently coded "1" and " 0 " respectively). An interaction between group and time was also included. Time was coded 0 at posttest so that the intercept represents the outcome at posttest and the main effect for group represents group differences at posttest. The group by time interaction, with time coded in months since posttest, represents group differences in change between posttest and 6-month follow-up. For the Minding Health vs. Healthy Weight group comparison baseline measures of eating disorder symptom scores were included as a covariate.

\section{Results}

Table 1 provides average means and standard deviations for the 20 data sets. Results of the random effects growth models for the Minding Health versus video controls are presented in Table 2. There was a significant group effect, driven by the fact that Minding Health participants showed significantly greater pre-post decreases in body fat than controls who increased in body fat over time; the marginal group by time interaction indicated that this effects faded by 6-month follow-up. There were no intervention effects for BMI, percent of calories from fat and sugar, or eating disorder symptoms.

Results of the Minding Health versus Healthy Weight comparison are presented in Table 3. There was a significant group effect for BMI, which was driven by a greater increase in BMI in Minding Health versus Healthy Weight participants from pre- to post and from pre- to 6month follow-up. There was a significant group and group-by-time effect for eating disorder symptoms, which was driven by greater decreases in symptoms in Healthy Weight versus Minding Health participants, with this effect being larger at post than at 6-month follow-up. There was a significant group effect for percent of calories from fat and sugar, driven by a greater decrease in Minding Health versus Healthy Weight participants from pre- to post, with this effect maintaining at the 6-month follow-up. There were no effects for body fat.

Results of the Healthy Weight versus video control comparisons are presented in Table 4. There was a significant group effect for percent body fat, driven by the fact that Healthy Weight participants showed a significantly greater pre-post decrease in body fat than controls who increased in body fat over time. There was a significant group effect for eating disorder symptoms, which was driven by greater pre-post decreases in symptoms in Healthy Weight versus control participants. There were no effects for BMI or percent of calories from fat and sugar.

Minding Health participants showed greater pre-post BOLD response increases in the left hemisphere in the insula, IFG, and middle temporal gyrus (MTG) in response to pictrures of high-fat/high-sugar food versus vegetables compared to controls (Table 5). Minding Health participants also showed greater pre-post BOLD response increases in bilateral MTG, superior temporal gyrus (STG), and insula, right IFG (Fig 3A), and left inferior parietal lobe in response to pictures of high-fat/high-sugar food versus water glasses compared to controls (Table 5). Minding Health participants showed greater pre-post BOLD response decreases in the left mid cingulate gyrus (Fig 3B) and right insula in response to the cue predicting 
milkshake receipt versus the cue predicting tasteless solution receipt compared to controls (Table 4). No significant effects were found for the milkshake receipt > tasteless solution receipt contrast.

\section{Discussion}

Minding Health versus control participants showed greater decreases in body fat, though this effect faded by 6-month follow-up. Minding Health versus Healthy Weight participants also reported reducing intake of dietary fat and sugar. However, Minding Health participants did not show greater improvements in BMI or eating disorder symptoms relative to control participants. Further, Minding Health versus Healthy Weight participants showed larger increases in BMI and smaller reductions in eating disorder symptoms. Minding Health participants did not show differential change in body fat relative to Healthy Weight participants. Further, Healthy Weight participants showed significantly greater decreases in body fat and eating disorder symptoms than controls. Given that Healthy Weight has produced significant reductions in BMI and eating disorder symptoms, and has significantly reduced onset of eating disorders over 2-3 year follow-ups in other trials $(45,53)$, it is perhaps not surprising that the new obesity program did not significantly outperform this established program on these outcomes. Indeed, the significantly greater weight gain and eating disorder prevention effects observed in Healthy Weight versus Minding Health participants, which are clinically meaningful medium sized effects, are noteworthy, as very few obesity or eating disorder prevention programs have been found to significantly outperform an alternative active intervention.

With regard to potential changes in neural responsivity, data provided evidence that after completing Minding Health participants showed stronger recruitment of a region implicated in inhibitory control (IFG) relative to control participants when exposed to images of highfat/high-sugar foods versus glasses of water; groups did not differ in activation in this region at pretest. Minding Health participants also showed a weaker recruitment of the left mid cingulate cortex, a region implicated in attention and expectation (54), at posttest; groups did not differ in activation in this region at pretest. The evidence that Minding Health participants showed increased recruitment of the IFG after reappraisal training dovetails with results from previous cognitive reappraisal studies $(23,24,27)$. Minding Health participants also showed stronger activation in bilateral insula, MTG, and STG at posttest relative to controls when exposed to images of high-fat/high-sugar foods versus vegetables and glasses of water at post. The insula has been shown to be involved in the processing of taste information (55) and interoceptive states such as hunger (56). The MTG and STG are involved in cognitive processes, including semantic memory (57). Perhaps the Minding Health participants were more focused on the specific qualities of the high-fat/high-sugar foods, including taste and on their interoceptive states. Again, participants were not asked to apply cognitive reappraisals during the scans.

It is important to consider the study limitations. First, the sample size was relatively small for the fMRI scans, increasing risk for false negative findings. Second, it would have been ideal if Healthy Weight participants had completed pre and post fMRI scans, as this alternative obesity prevention program was more structurally parallel to the Minding Health 
obesity prevention program in terms of therapist contact and duration. Third, although we expressly asked Minding Health participants to use the cognitive reappraisals between sessions, we did not attempt to assess the frequency of reappraisal usage. Fourth, because self-report measures of dietary intake are biased, it would have been preferable if an objective measure of this outcome had been included.

In sum, the evidence that Minding Health participants showed reductions in body fat relative to controls and in reported fat and sugar intake relative to Healthy Weight participants is encouraging, but the lack of effects for BMI, and the superiority of the intervention effects for the Healthy Weight intervention implies there is room for improvement in the use of reappraisal training for weight gain prevention. Results also suggests that cognitive reappraisal training might be useful in helping participants increase recruitment of inhibitory regions when confronted with high-fat/high-sugar foods, though there was little evidence that this training reduced reward region response to such foods, implying that it would be useful to improve the training procedures and consider supplementing the training with additional interventions. For instance, it might be beneficial to use real time fMRI biofeedback to help participants learn how to optimally reduce reward region activation and increase inhibitory region activation when encountering high-fat/high-sugar foods, as preliminary evidence suggests that this technique was effective (27). The current findings did not provide support for the utility of the palate-retraining element of the intervention, which suggests that it might be best to drop this element of the intervention and focus more on enhancing training in the use of cognitive reappraisals. We are hopeful that with continued refinement, cognitive reappraisal training might prove useful in preventing and treating obesity, which has been illusive with extant interventions.

\section{Acknowledgments}

This work was supported by National Institute of Child Health \& Human Development grant (R01 HD071900, 07/01/12-06.30/17). The authors thank the Lewis Center for Neuroimaging at the University of Oregon for their assistance in data collection for these projects.

\section{References}

1. Flegal KM, Carroll MD, Kit BK, Ogden CL. Prevalence of obesity and trends in the distribution of body mass index among US adults, 1999-2010. JAMA. 2012; 307:491-497. [PubMed: 22253363]

2. Harris KC, Kuramoto LK, Schulzer M, Retallack JE. Effect of school-based physical activity interventions on body mass index in children: a meta-analysis. CAMJ. 2009; 180:719-726.

3. Martin LE, Holsen LM, Chambers RJ, Bruce AS, Brooks WM, Zarcone JR, et al. Neural mechanisms associated with food motivation in obese and healthy weight adults. Obesity. 2010; 18:254-260. [PubMed: 19629052]

4. Rothemund Y, Preuschhof C, Bohner G, Bauknecht HC, Klingebiel R, Flor H, et al. Differential activation of the dorsal striatum by high-calorie visual food stimuli in obese individuals. NeuroImage. 2007; 37:410-421. [PubMed: 17566768]

5. Stice E, Yokum S, Bohon C, Marti N, Smolen A. Reward circuitry responsivity to food predicts future increases in body mass: moderating effects of DRD2 and DRD4. NeuroImage. 2010; 50:1618-1625. [PubMed: 20116437]

6. Stoeckel LE, Weller RE, Cook EW, Twieg DB, Knowlton RC, Cox JE. Widespread reward-system activation in obese women in response to pictures of high-calorie foods. NeuroImage. 2008; 41:636-647. [PubMed: 18413289] 
7. Chouinard-Decorte FFJ, Small D. Increased amygdala response and decreased influence of internal state on amygdala response to food in overweight compared to healthy weight individuals. Appetite. 2010; 54:639.

8. Demos KE, Heatherton TF, Kelley WM. Individual differences in nucleus accumbens activity to food and sexual images predict weight gain and sexual behavior. J Neurosci. 2012; 32:5549-5552. [PubMed: 22514316]

9. Yokum S, Ng J, Stice E. Attentional bias to food images associated with elevated weight and future weight gain: an fMRI study. Obesity. 2011; 19:1775-1783. [PubMed: 21681221]

10. Stice E, Spoor S, Bohon C, Veldhuizen MG, Small DM. Relation of reward from food intake and anticipated food intake to obesity: a functional magnetic resonance imaging study. J Abnorm Psychol. 2008; 117:924-935. [PubMed: 19025237]

11. Berridge KC. 'Liking' and 'wanting' food rewards: brain substrates and roles in eating disorders. Physiolo Behav. 2009; 97:537-550.

12. Batterink L, Yokum S, Stice E. Body mass correlates inversely with inhibitory control in response to food among adolescent girls: an fMRI study. NeuroImage. 2010; 52:1696-1703. [PubMed: 20510377]

13. Nummenmaa L, Hirvonen J, Hannukainen JC, Immonen H, Lindroos MM, Salminen P, et al. Dorsal striatum and its limbic connectivity mediate abnormal anticipatory reward processing in obesity. PloS One. 2012; 7:e31089. [PubMed: 22319604]

14. Bruce AS, Lepping RJ, Bruce JM, Cherry JB, Martin LE, Davis AM, et al. Brain responses to food logos in obese and healthy weight children. J Pediatr. 2013; 162:759-764e2. [PubMed: 23211928]

15. Epstein LH, Dearing KK, Temple JL, Cavanaugh MD. Food reinforcement and impulsivity in overweight children and their parents. Eat Beh. 2008; 9:319-327.

16. Nederkoorn C, Smulders FT, Havermans RC, Roefs A, Jansen A. Impulsivity in obese women. Appetite. 2006; 47:253-256. [PubMed: 16782231]

17. Rasmussen EB, Lawyer SR, Reilly W. Percent body fat is related to delay and probability discounting for food in humans. Behav Processes. 2010; 83:23-30. [PubMed: 19744547]

18. Cornier MA, Salzberg AK, Endly DC, Bessesen DH, Tregellas JR. Sex-based differences in the behavioral and neuronal responses to food. Physiol Behav. 2010; 99:538-543. [PubMed: 20096712]

19. Kishinevsky FI, Cox JE, Murdaugh DL, Stoeckel LE, Cook EW, Weller RE. fMRI reactivity on a delay discounting task predicts weight gain in obese women. Appetite. 2012; 58:582-592. [PubMed: 22166676]

20. Seeyave DM, Coleman S, Appugliese D, Corwyn RF, Bradley RH, Davidson NS, et al. Ability to delay gratification at age 4 years and risk of overweight at age 11 years. Arch Pediatr Adolesc Med. 2009; 163:303-308. [PubMed: 19349558]

21. Sutin AR, Ferrucci L, Zonderman AB, Terracciano A. Personality and obesity across the adult life span. J Pers Soc Psychol. 2011; 101:579-592. [PubMed: 21744974]

22. Pauli-Pott U, Albayrak O, Hebebrand J, Pott W. Does inhibitory control capacity in overweight and obese children and adolescents predict success in a weight-reduction program? Eur Child Adolesc Psychiatry. 2010; 19:135-141. [PubMed: 19644731]

23. Hollmann M, Hellrung L, Pleger B, Schlogl H, Kabisch S, Stumvoll M, et al. Neural correlates of the volitional regulation of the desire for food. Int J Obes. 2012; 36:648-655.

24. Kober H, Mende-Siedlecki P, Kross EF, Weber J, Mischel W, Hart CL, et al. Prefrontal-striatal pathway underlies cognitive regulation of craving. Proc Natl Acad Sci U S A. 2010; 107:1481114816. [PubMed: 20679212]

25. Siep N, Roefs A, Roebroeck A, Havermans R, Bonte M, Jansen A. Fighting food temptations: the modulating effects of short-term cognitive reappraisal, suppression and up-regulation on mesocorticolimbic activity related to appetitive motivation. NeuroImage. 2012; 60:213-220. [PubMed: 22230946]

26. Yokum S, Stice E. Cognitive regulation of food craving: effects of three cognitive reappraisal strategies on neural response to palatable foods. Int J Obes. 2013; 37:1565-1570. 
27. Stoeckel, L.; Ghosh, S.; Stern, J.; Keshavan, A.; Gabrieli, J.; Whitfield-Gabrieli; Evins, A. Real time fMRI neurofeedback: Effect on food and cigarette cue reactivity. Paper presented at the American College of Neuropsychopharmacology; Florida. 2013.

28. Halford JC, Boyland EJ, Hughes GM, Stacey L, McKean S, Dovey TM. Beyond-brand effect of television food advertisements on food choice in children: the effects of weight status. Public Health Nutr. 2008; 11:897-904. [PubMed: 18005487]

29. de Weijer BA, van de Giessen E, van Amelsvoort TA, Boot E, Braak B, Janssen IM, et al. Lower striatal dopamine D2/3 receptor availability in obese compared with non-obese subjects. EJNMMI Res. 2011; 1:37. [PubMed: 22214469]

30. Volkow ND, Wang GJ, Telang F, Fowler JS, Thanos PK, Logan J, et al. Low dopamine striatal D2 receptors are associated with prefrontal metabolism in obese subjects: possible contributing factors. NeuroImage. 2008; 42:1537-1543. [PubMed: 18598772]

31. Frank GK, Reynolds JR, Shott ME, Jappe L, Yang TT, Tregellas JR, et al. Anorexia nervosa and obesity are associated with opposite brain reward response. Neuropsychopharmacology. 2012; 37:2031-2046. [PubMed: 22549118]

32. Green E, Jacobson A, Haase L, Murphy C. Reduced nucleus accumbens and caudate nucleus activation to a pleasant taste is associated with obesity in older adults. Brain Res. 2011; 1386:109117. [PubMed: 21362414]

33. Stice E, Spoor S, Bohon C, Small DM. Relation between obesity and blunted striatal response to food is moderated by TaqIA A1 allele. Science. 2008; 322:449-452. [PubMed: 18927395]

34. Stice E, Yokum S, Blum K, Bohon C. Weight gain is associated with reduced striatal response to palatable food. J Neurosci. 2010; 30:13105-13109. [PubMed: 20881128]

35. Davis JF, Tracy AL, Schurdak JD, Tschop MH, Lipton JW, Clegg DJ, et al. Exposure to elevated levels of dietary fat attenuates psychostimulant reward and mesolimbic dopamine turnover in the rat. Behav Neurosci. 2008; 122:1257-1263. [PubMed: 19045945]

36. Johnson PM, Kenny PJ. Dopamine D2 receptors in addiction-like reward dysfunction and compulsive eating in obese rats. Nat Neurosci. 2010; 13:635-641. [PubMed: 20348917]

37. Alsio J, Olszewski PK, Norback AH, Gunnarsson ZE, Levine AS, Pickering C, et al. Dopamine D1 receptor gene expression decreases in the nucleus accumbens upon long-term exposure to palatable food and differs depending on diet-induced obesity phenotype in rats. Neuroscience. 2010; 171:779-787. [PubMed: 20875839]

38. Ledikwe JH, Ello-Martin J, Pelkman CL, Birch LL, Mannino ML, Rolls BJ. A reliable, valid questionnaire indicates that preference for dietary fat declines when following a reduced-fat diet. Appetite. 2007; 49:74-83. [PubMed: 17275138]

39. Mattes RD. Fat preference and adherence to a reduced-fat diet. Am J Clin Nutr. 1993; 57:373-381. [PubMed: 8438771]

40. Stewart JE, Feinle-Bisset C, Golding M, Delahunty C, Clifton PM, Keast RS. Oral sensitivity to fatty acids, food consumption and BMI in human subjects. Br J Nutr. 2010; 104:145-152. [PubMed: 20196892]

41. Lowe MR, Annunziato RA, Markowitz JT, Didie E, Bellace DL, Riddell L, et al. Multiple types of dieting prospectively predict weight gain during the freshman year of college. Appetite. 2006; 47:83-90. [PubMed: 16650913]

42. Weyers AM, Mazzetti SA, Love DM, Gomez AL, Kraemer WJ, Volek JS. Comparison of methods for assessing body composition changes during weight loss. Med Sci Sports Exerc. 2002; 34:497502. [PubMed: 11880815]

43. Cole TJ, Faith MS, Pietrobelli A, Heo M. What is the best measure of adiposity change in growing children: BMI, BMI \%, BMI z-score or BMI centile? Eur J Clin Nutr. 2005; 59:419-425.

[PubMed: 15674315]

44. Pietrobelli A, Faith MS, Allison DB, Gallagher D, Chiumello G, Heymsfield SB. Body mass index as a measure of adiposity among children and adolescents: a validation study. J Pediatr. 1998; 132:204-210. [PubMed: 9506629]

45. Stice E, Rohde P, Shaw H, Marti CN. Efficacy trial of a selective prevention program targeting both eating disorders and obesity among female college students: 1- and 2-year follow-up effects. J Consult Clin Psychol. 2013; 81:183-189. [PubMed: 23231574] 
46. Block G, Subar AF. Estimates of nutrient intake from a food frequency questionnaire: the 1987 National Health Interview Survey. J Am Diet Assoc. 1992; 92:969-977. [PubMed: 1640041]

47. Surrao J, Sawaya AL, Dallal GE, Tsay R, Roberts SB. Use of food quotients in human doubly labeled water studies: comparable results obtained with 4 widely used food intake methods. J Am Diet Assoc. 1998; 98:1015-1020. [PubMed: 9739802]

48. Klohe DM, Clarke KK, George GC, Milani TJ, Hanss-Nuss H, Freeland-Graves J. Relative validity and reliability of a food frequency questionnaire for a triethnic population of 1-year-old to 3-year-old children from low-income families. J Am Diet Assoc. 2005; 105:727-734. [PubMed: 15883549]

49. Stice E, Yokum S, Burger KS, Epstein LH, Small DM. Youth at risk for obesity show greater activation of striatal and somatosensory regions to food. J Neurosci. 2011; 31:4360-4366. [PubMed: 21430137]

50. Graham JW. Missing data analysis: making it work in the real world. Annu Rev Psychol. 2009; 60:549-576. [PubMed: 18652544]

51. Raghunathan, TE.; Solenberger, PW.; Van Hoewyk, J. IVEware: imputation and variance estimation software. Ann Arbor, MI: Institute for Social Research. University of Michigan; 2002.

52. Rubin, DB. Multiple Imputation for Nonresponse in Surveys. New York: John Wiley \& Sons, Inc; 1987.

53. Stice E, Marti CN, Spoor S, Presnell K, Shaw H. Dissonance and healthy weight eating disorder prevention programs: long-term effects from a randomized efficacy trial. J Consult Clin Psychol. 2008; 76:329-340. [PubMed: 18377128]

54. Stevens FL, Hurley RA, Taber KH. Anterior cingulate cortex: unique role in cognition and emotion. J Neuropsychiatry Clin Neurosci. 2011; 23:121-125. [PubMed: 21677237]

55. Bermudez-Rattoni F. The forgotten insular cortex: Its role on recognition memory formation. Neurobiol Learn Mem. 2014; 109C:207-216. [PubMed: 24406466]

56. Haase L, Cerf-Ducastel B, Murphy C. Cortical activation in response to pure taste stimuli during the physiological states of hunger and satiety. NeuroImage. 2009; 44:1008-1021. [PubMed: 19007893]

57. Visser M, Jefferies E, Embleton KV, Lambon Ralph MA. Both the middle temporal gyrus and the ventral anterior temporal area are crucial for multimodal semantic processing: distortion-corrected fMRI evidence for a double gradient of information convergence in the temporal lobes. J Cogn Neurosci. 2012; 24:1766-1778. [PubMed: 22621260] 


\section{Highlights (3-5 bullets; max 85 characters, including spaces per bullet)}

- High reward response and low inhibitory response to food may drive overeating.

- We tested an obesity prevention program focused using cognitive reappraisals

- Reappraisals aimed to reduce reward activation \& increase inhibitory activation

- We assessed neural response to food, BMI \& body fat in an RCT over 6-months

- The reappraisal program resulted in short-term decreases in fat \& neural changes 


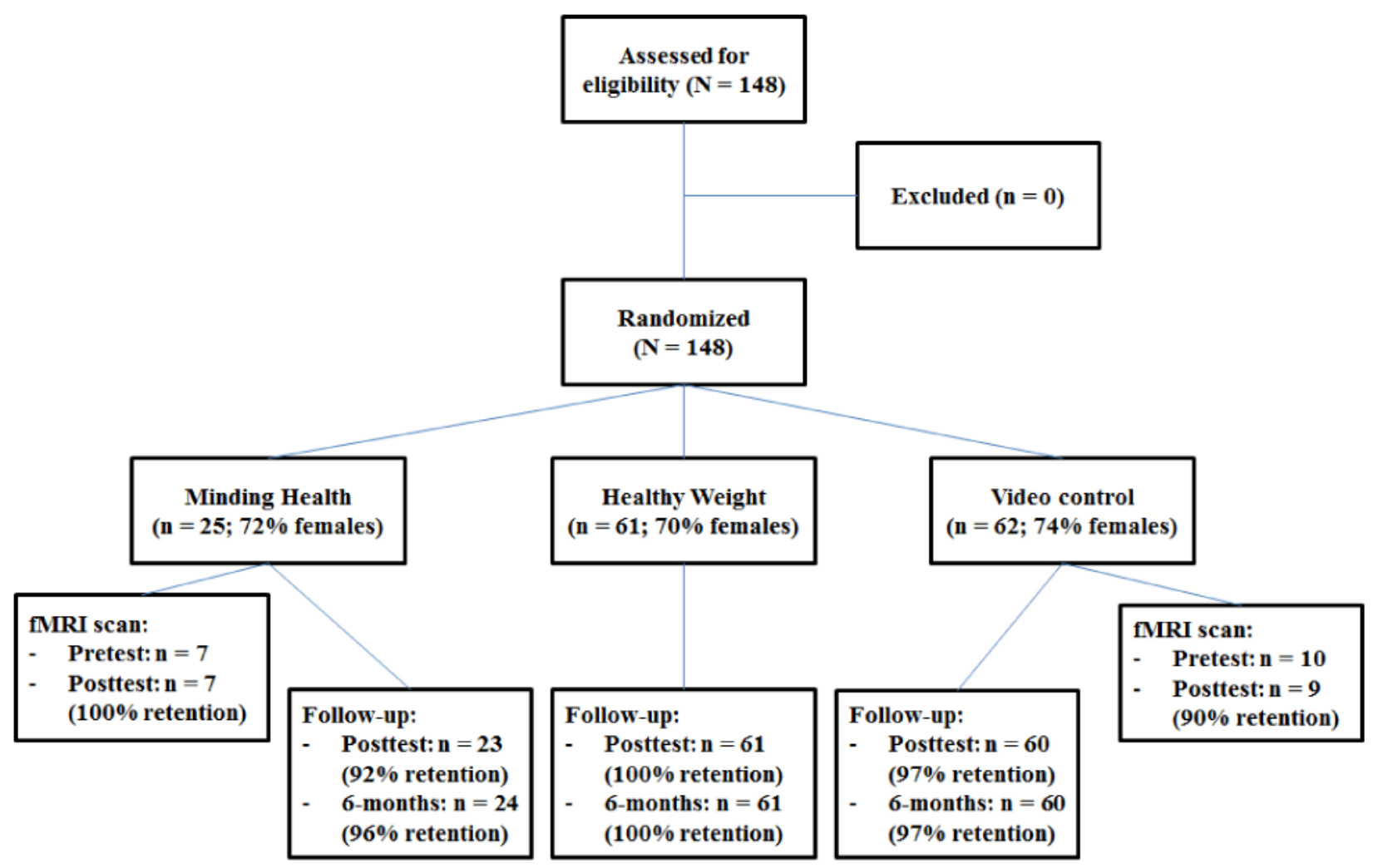

Figure 1. Participant flow information 

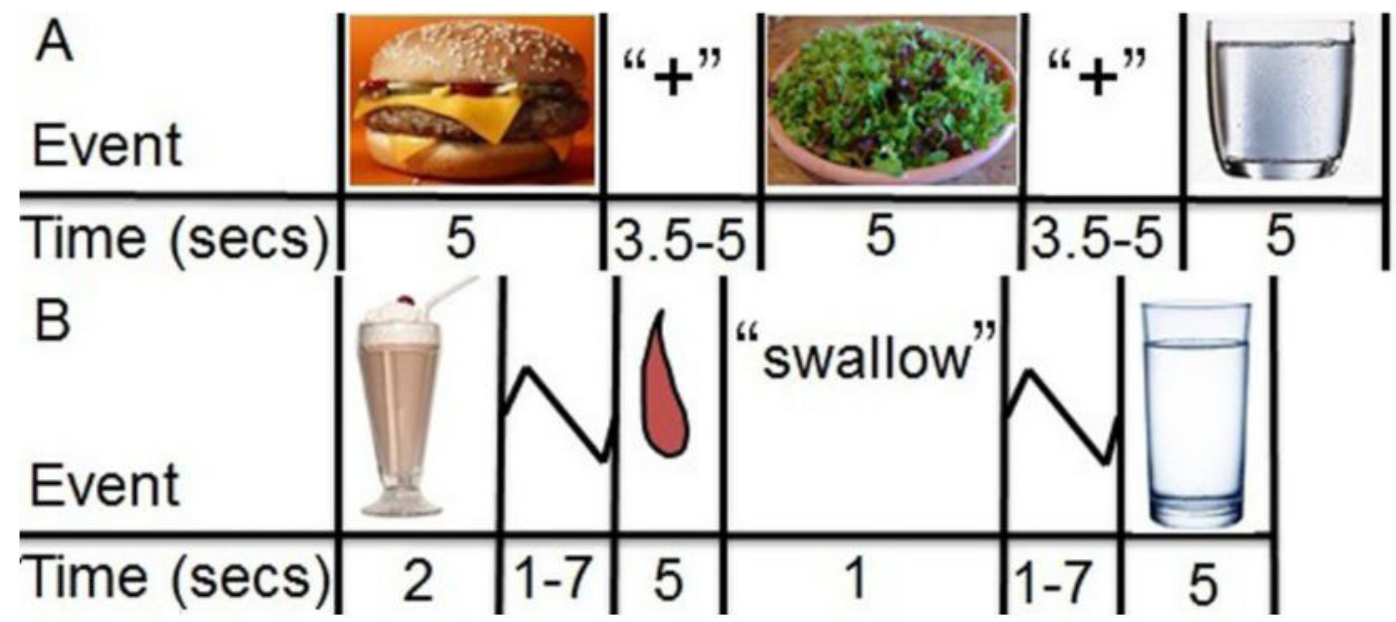

Figure 2.

Timing and ordering of presentation of events during A) the food picture paradigm and B) the milkshake paradigm. 

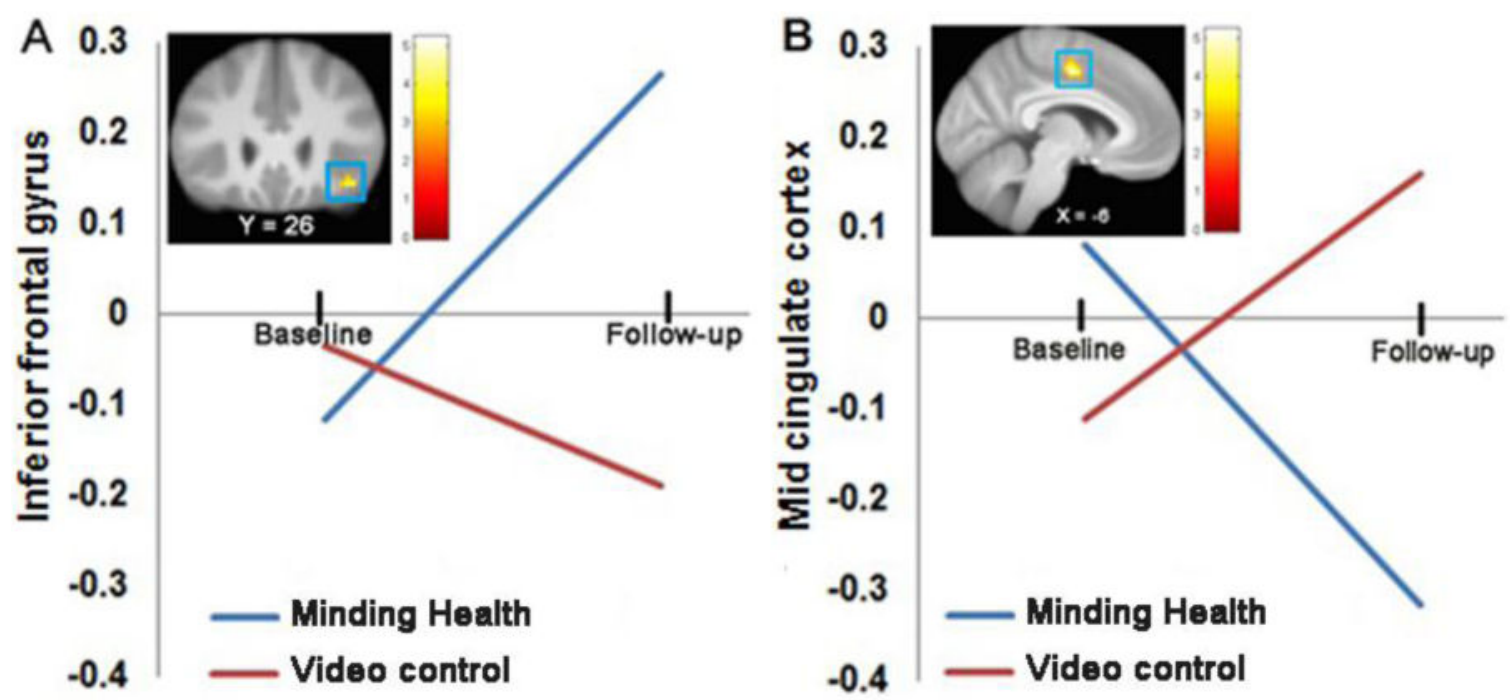

Figure 3.

A. Greater baseline to post BOLD response increases in the right inferior frontal gyrus (MNI coordinates: $39,26,-14, \mathrm{Z}=3.22$, cluster size $(k): 28 ; r=0.81)$ in response to images of high-fat/high-sugar food relative to glasses of water in the intervention group relative to the control group. B. Greater baseline to post BOLD response decreases in the left mid cingulate cortex (MNI coordinates: $-6,-10,46, \mathrm{Z}=4.29, k=30 ; r>0.9$ ) in response to milkshake cue relative to tasteless solution cue in the intervention group relative to the control group. 


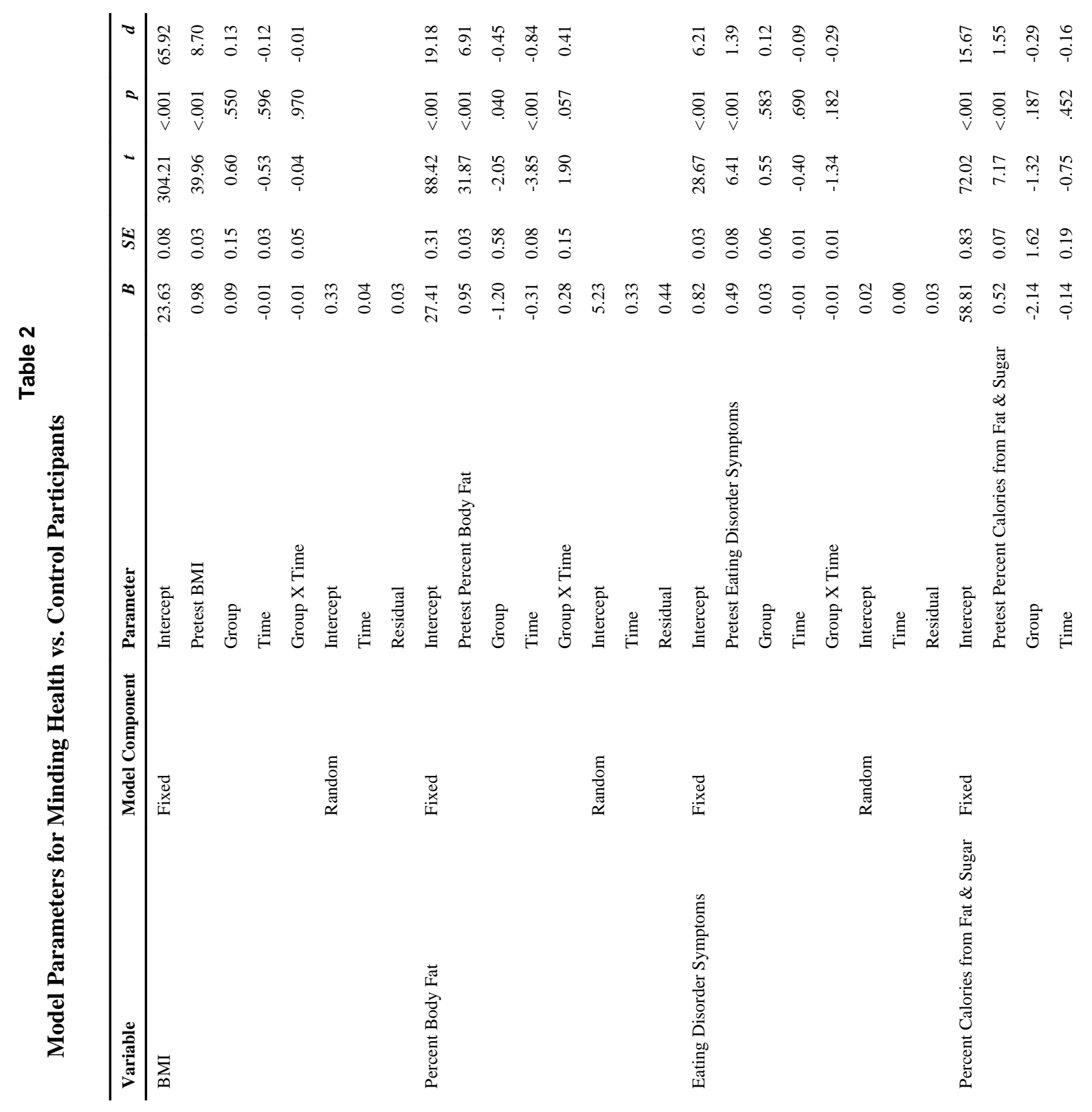




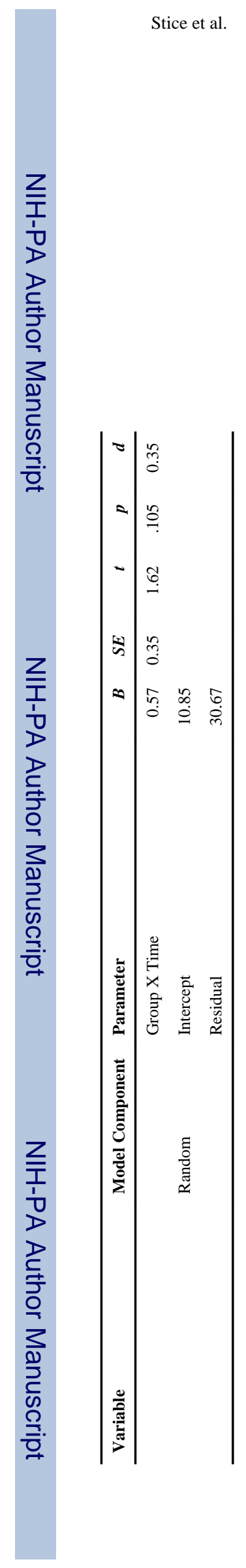

Page 20

Physiol Behav. Author manuscript; available in PMC 2016 January 01. 


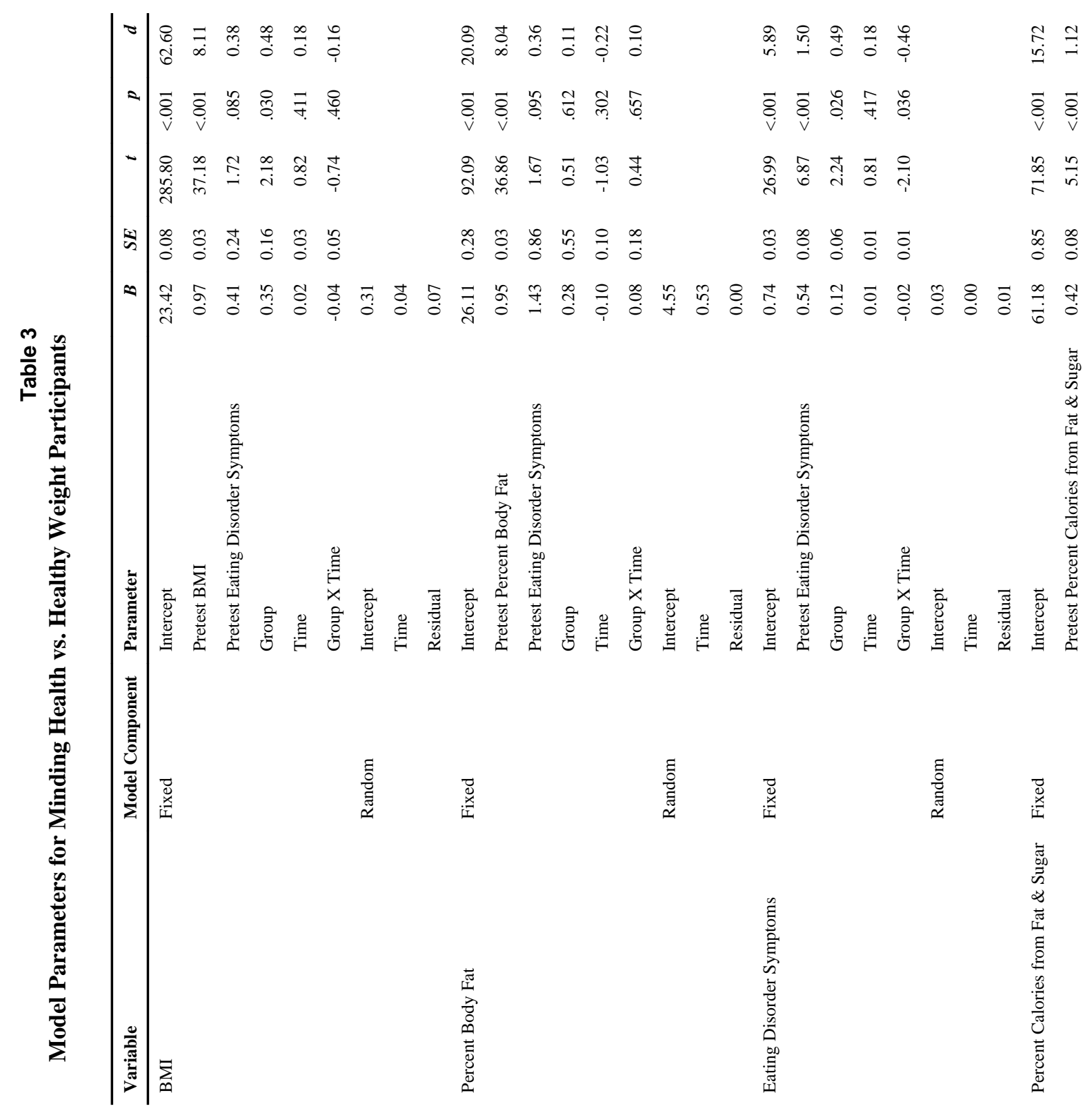




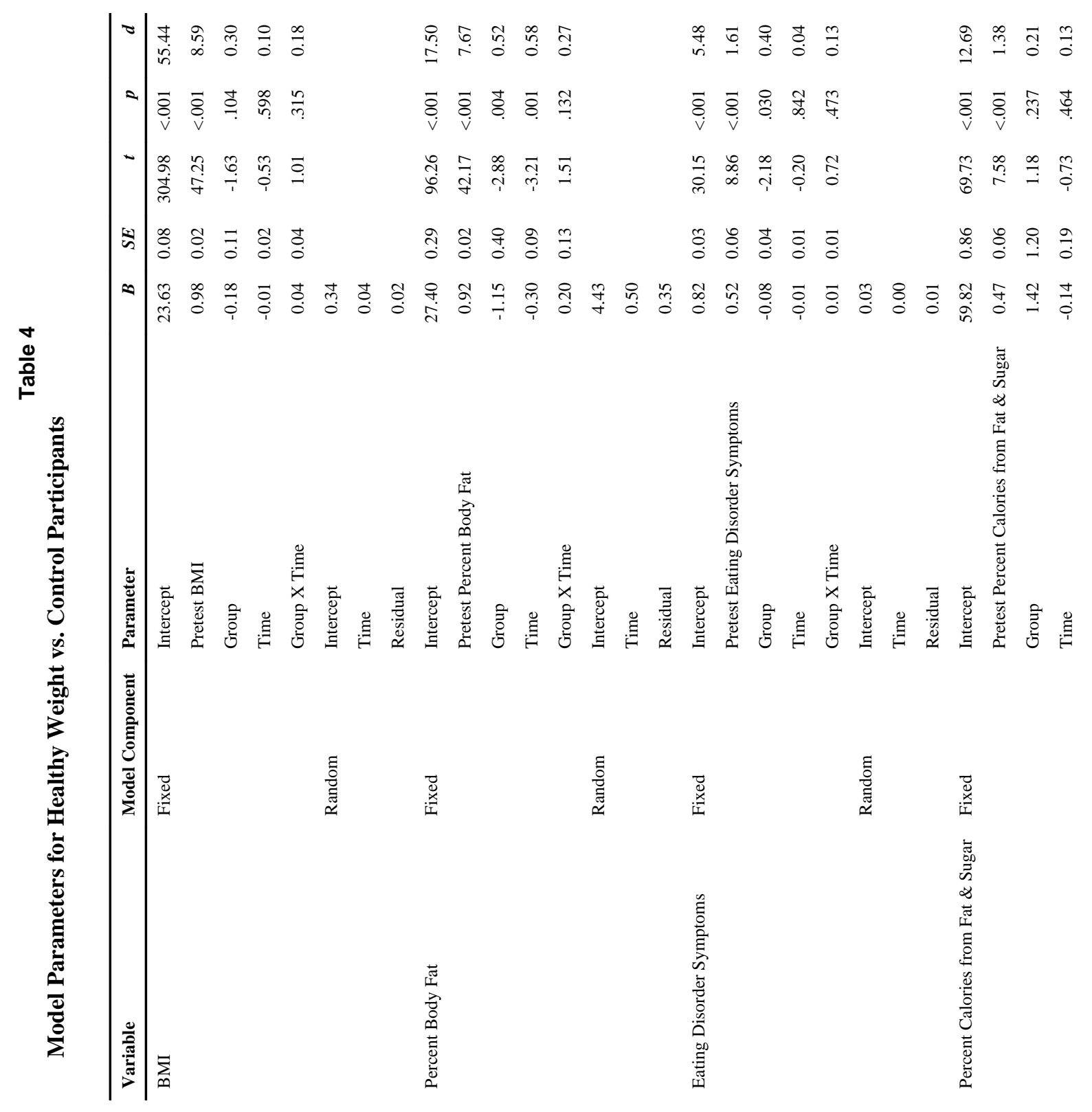




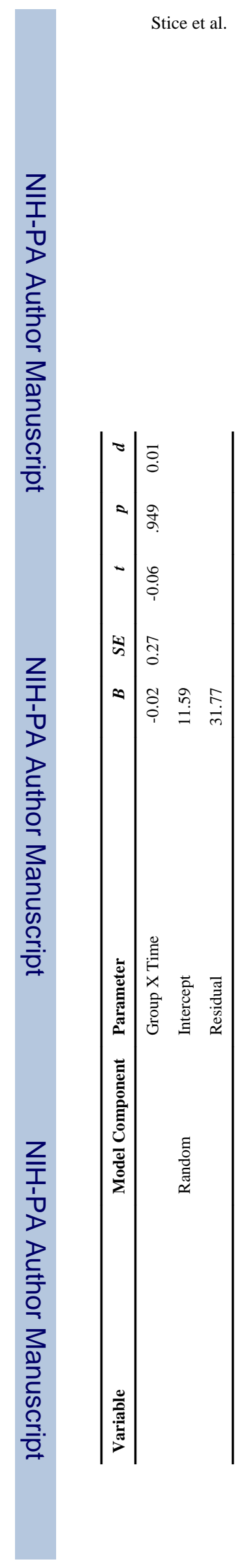

Page 24

Physiol Behav. Author manuscript; available in PMC 2016 January 01. 


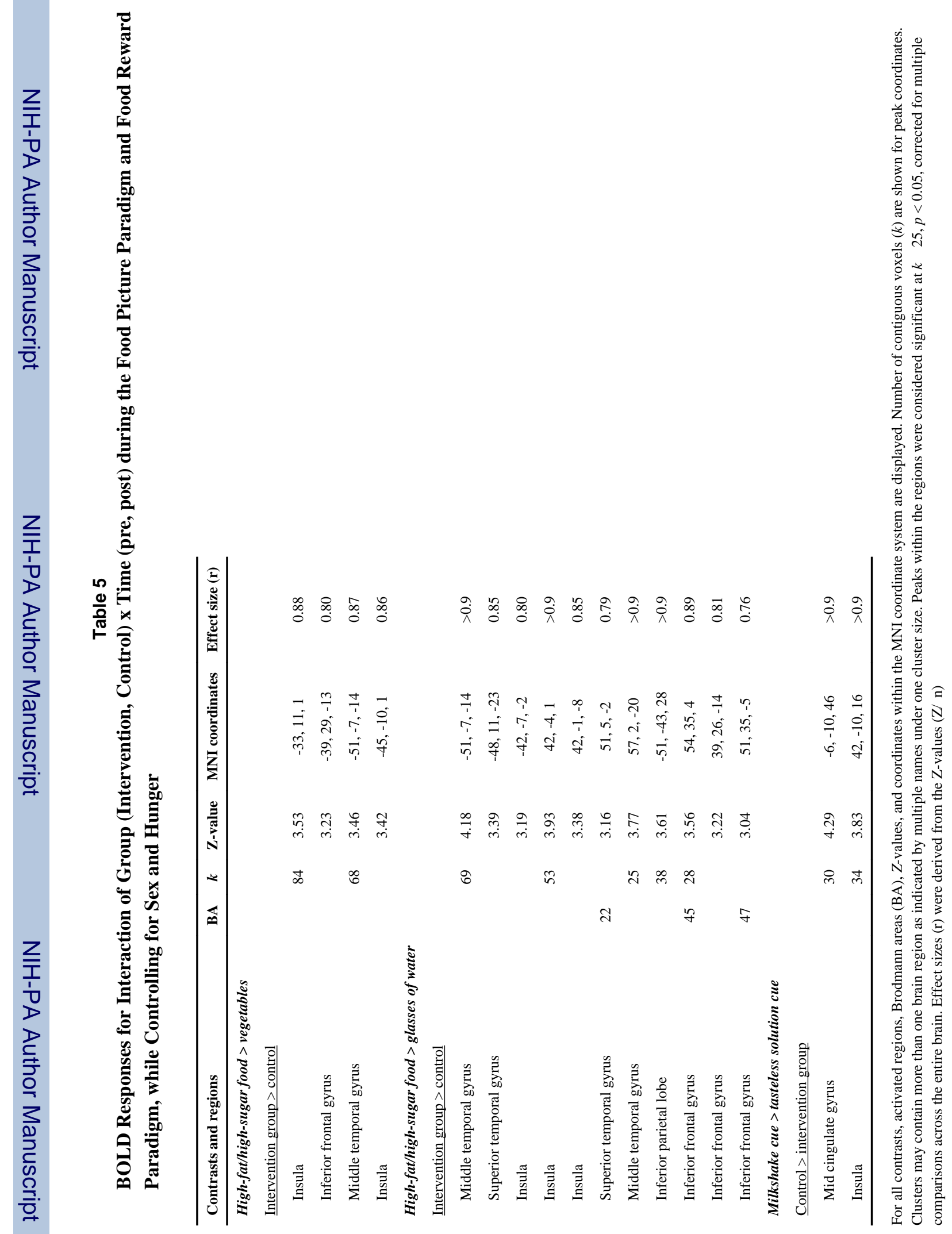

Physiol Behav. Author manuscript; available in PMC 2016 January 01. 